EPiC Series in Engineering
Volume 3, 2018, Pages 2268-2275
HIC 2018. 13th International
Conference on Hydroinformatics

\title{
A 3D hydrodynamic model for shallow water flow through a circular patch of emergent cylinders
}

\author{
Jian Wang ${ }^{1}$, Lei $\mathrm{Li}^{2}$, Jingxin Zhang ${ }^{1}$, Dongfang Liang ${ }^{1}$ and Qi Yang ${ }^{1}$ \\ ${ }^{1}$ MOE Key Laboratory of Hydrodynamics, Shanghai Jiao Tong University, Shanghai 200240, \\ China \\ ${ }^{2}$ Department of Naval Architecture, Ocean \& Marine Engineering University of Strathclyde, \\ Glasgow G4 OLZ, UK \\ lei.li@strath.ac.uk
}

\begin{abstract}
3D numerical computations are performed to simulate the shallow water flow through an array of non-submerged cylinders occupying a circular area in the middle of the domain. A hydrodynamic model capable of capturing the free surface positions is developed with the SST (shear strain transport) k- $\omega$ turbulence closure. The model is first verified and validated against experimental data available in the literature. It is demonstrated that the present model can predict both the average velocity and turbulence structure well. In addition, both cylinder-scale flow as well as patch-scale flow can be well reproduced. The velocity field and distribution of bed stress are then analyzed to study the flow patterns and sediment deposition with different solid volume fractions and water depths.
\end{abstract}

\section{Introduction}

Aquatic vegetation plays a significant role in natural channels because of its influence on hydraulics and ecosystem. It can change the distribution of flow velocity, turbulence structure and patterns of deposition and erosion. Vegetation also provides a broader range of ecosystem services. Water quality can be improved by the production of oxygen and the uptake of nutrients. The removal of inorganic substances such as nitrogen and phosphorous are also strengthened. Also, vegetation plays a crucial role in forming the foundation of the food chain, and biodiversity in channels is promoted by creating different habitats. Therefore, a variety of experiments and numerical simulations have been carried out to investigate the flow structures, drag and sediment transport characteristics in flow with vegetation. 
Nezu and Onitsuka [1] and Ghisalberti and Nepf [2] conducted a series of experiments to research the flow patterns, turbulence and diffusion in shallow water flow with vegetation. In these studies, the vegetation elements are simplified as rigid bodies, and the vegetation zone is regarded as an array of solid cylinders [3].

As for the numerical models, the 2D shallow water model is widely used for simulating flow through vegetation. $\mathrm{Yu}$, et al. [4] used two 2D models (the multi-body model and porous model) coupled with a scale adaptive simulation (SAS) turbulence model to simulate flow through a circular patch of emergent cylinders. Their computed results agree well with the experimental data regarding the mean velocity $\bar{u}$ and $\bar{v}$, but fail to predict the magnitude of the turbulent velocity. The additional effect of 3D turbulent vortex dissipation and stretching can affect the flow significantly, which is neglected in the 2D model. The flow around a single cylinder is also three-dimensional and cannot be accurately reproduced. Moreover, the free surface and bed shear friction are not always included in the $2 \mathrm{D}$ model. When the patch diameter $D$ is large, and the water depth $H$ is low, the bed friction becomes significant enough to suppress the vortex street and could not be ignored[5]. Then the flow will become three-dimensional, and a robust 3D model should be considered. In this paper, a 3D nonhydrostatic FVM model based on unstructured grids is developed and used to simulate shallow water flow through a circular array of non-submerged cylinders. Furthermore, the flow patterns and bed shear stress are compared between cases with different solid volume fractions and water depths.

\section{Numerical model}

\subsection{Governing equations}

In the present mathematical model, the total pressure is represented as a superposition of the hydrostatic component $p=\rho g(\eta-z)$ and the non-hydrostatic component $q$ [6]. Considering the effect of free surface and bed friction, we transform the vertical coordinate $z$ to the $\sigma$ coordinate[7], and the modified equations can be rewritten as:

$$
\begin{gathered}
\frac{\partial \eta}{\partial t}+\frac{\partial H u}{\partial x}+\frac{\partial H v}{\partial y}+\frac{\partial H w_{\sigma}}{\partial \sigma}=0 \\
\frac{\partial H u}{\partial t}+\frac{\partial H u u}{\partial x}+\frac{\partial H u v}{\partial y}+\frac{\partial H u w_{\sigma}}{\partial \sigma}=-g H \frac{\partial \eta}{\partial x}-\frac{H}{\rho} \frac{\partial q}{\partial x}+\frac{\partial}{\partial x}\left(v_{t} \frac{\partial H u}{\partial x}\right)+\frac{\partial}{\partial y}\left(v_{t} \frac{\partial H u}{\partial y}\right)+\frac{1}{H} \frac{\partial}{\partial \sigma}\left(\frac{v_{t}}{H} \frac{\partial H u}{\partial \sigma}\right) \\
\frac{\partial H v}{\partial t}+\frac{\partial H v u}{\partial x}+\frac{\partial H v v}{\partial y}+\frac{\partial H v w_{\sigma}}{\partial \sigma}=-g H \frac{\partial \eta}{\partial y}-\frac{H}{\rho} \frac{\partial q}{\partial y}+\frac{\partial}{\partial x}\left(v_{t} \frac{\partial H v}{\partial x}\right)+\frac{\partial}{\partial y}\left(v_{t} \frac{\partial H v}{\partial y}\right)+\frac{1}{H} \frac{\partial}{\partial \sigma}\left(\frac{v_{t}}{H} \frac{\partial H v}{\partial \sigma}\right) \\
\frac{\partial H w}{\partial t}+\frac{\partial H w u}{\partial x}+\frac{\partial H w v}{\partial y}+\frac{\partial H w w_{\sigma}}{\partial \sigma}=-\frac{H}{\rho} \frac{\partial q}{\partial \sigma}+\frac{\partial}{\partial x}\left(v_{t} \frac{\partial H w}{\partial x}\right)+\frac{\partial}{\partial y}\left(v_{t} \frac{\partial H w}{\partial y}\right)+\frac{1}{H} \frac{\partial}{\partial \sigma}\left(\frac{v_{t}}{H} \frac{\partial H w}{\partial \sigma}\right)
\end{gathered}
$$

where $\eta$ is the free surface elevation, $h$ is the still water depth, and $H=h+\eta$ is the total water depth. $u, v, w$ are the velocities in $x, y, z$ directions, respectively. $\sigma=(z-\eta) /(h+\eta), g$ is gravitational acceleration, $v_{t}$ is the eddy viscosity coefficient. $w_{\sigma}$ is the vertical velocity in $\sigma$ coordinate, and can be derived from:

$$
w_{\sigma}=\frac{w}{H}-\frac{u}{H}\left(\sigma \frac{\partial H}{\partial x}+\frac{\partial \eta}{\partial x}\right)-\frac{v}{H}\left(\sigma \frac{\partial H}{\partial y}+\frac{\partial \eta}{\partial y}\right)-\frac{1}{H}\left(\sigma \frac{\partial H}{\partial t}+\frac{\partial \eta}{\partial t}\right)
$$

\subsection{Turbulence model}

To close the system, the Shear-Stress Transport (SST) $k-\omega$ turbulence model is employed.

$$
\frac{\partial}{\partial t}(\rho k)+\frac{\partial}{\partial x_{i}}\left(\rho k u_{i}\right)=\frac{\partial}{\partial x_{j}}\left(\mathrm{~T}_{k} \frac{\partial k}{\partial x_{j}}\right)+G_{k}-Y_{k}, \quad(i, j=1,2,3)
$$




$$
\frac{\partial}{\partial t}(\rho \omega)+\frac{\partial}{\partial x_{j}}\left(\rho \omega u_{j}\right)=\frac{\partial}{\partial x_{j}}\left(\mathrm{~T}_{\omega} \frac{\partial \omega}{\partial x_{j}}\right)+G_{\omega}-Y_{\omega}+D_{\omega}
$$

Where $G_{k}$ and $G_{w}$ are the production terms, $Y_{k}$ and $Y_{w}$ are the dissipation terms, $T_{k}$ and $T_{w}$ are the diffusion coefficients with respect to $k$ and $w$, and $D_{w}$ is the orthogonal term. Values of related parameters can be found in [8].

\section{Computational domain}

The proposed model is validated by the experiments carried out by Zong and Nepf [5]. Their tests were conducted in a channel with a length of $13 \mathrm{~m}$ and a width of $1.2 \mathrm{~m}$. A circular patch of wooden cylinders was put in the middle of the channel in a staggered arrangement. The leading edge of the patch was $3 \mathrm{~m}$ away from the inlet. The diameter of the cylinders $d$, was $0.6 \mathrm{~cm}$, and the diameter of the patch $D$, was $22 \mathrm{~cm}$.

In the simulations, a fine mesh is used near the cylinder wall, and a coarse mesh is employed far away from the patch zone. Wall function is adopted on the cylinder wall and bed. Discharge boundary condition is imposed at the inlet, and water level boundary condition is imposed at the outlet. The time step for computation is $0.0005 \mathrm{~s}$, when the variation of recorded water level at a gauge is within $\pm 0.001 \mathrm{~m}$, the simulation is assumed to be stable. The computed results during a period of $60 \mathrm{~s}$ are then recorded and output, with the frequency being $20 \mathrm{~Hz}$.

Six cases were simulated in this study, with the solid volume fraction $\varphi$ being 0.03 and 0.10 , and the water depth $H$ varies from $0.0665 \mathrm{~m}, 0.133 \mathrm{~m}$ to $0.266 \mathrm{~m} . U_{\infty}=0.098 \mathrm{~m} / \mathrm{s}$, is the velocity at the inlet, and the cylinder Reynolds number, $R e_{d}=U d / v$ is 588 , where $U$ is the mean velocity.

\section{Model validation}

\subsection{Mean velocity profiles}

To validate the model, the computed mean velocity profiles $\bar{u}$ (at $Y=0$ ) and $\bar{v}$ (at $Y=D / 2$ ) of case $H=0.133 \mathrm{~m}$ are depicted and compared with experimental results in Figure 1 and Figure 2 respectively. The gap between the solid and dash line in Figure 1-2 represents the vegetation zone $(0$ $<x / D<1)$. In this region, experimental measurements are not available as the gap distance between cylinders is very small. In general, good agreements between the predicted and measured curves are observed, which demonstrates the validity of the present model.

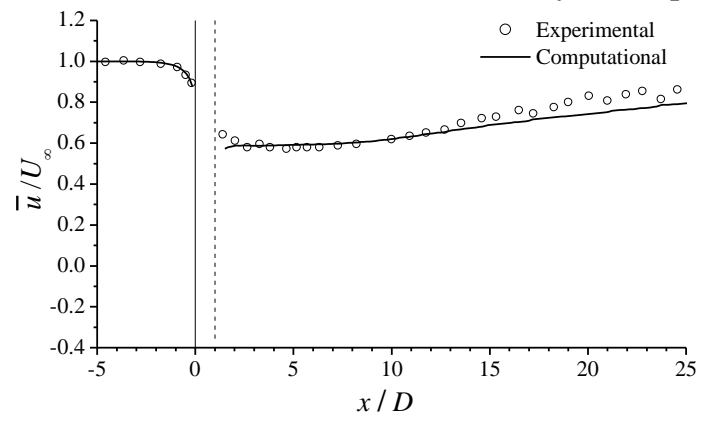

(a) $\varphi=0.03, H=0.133 \mathrm{~m}$

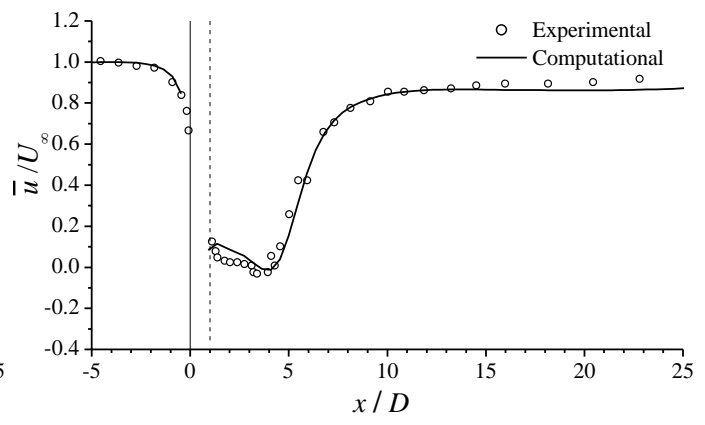

(b) $. \varphi=0.10, H=0.133 \mathrm{~m}$

Figure 1 Mean velocity profiles: along centreline $\mathrm{Y}=0$ 


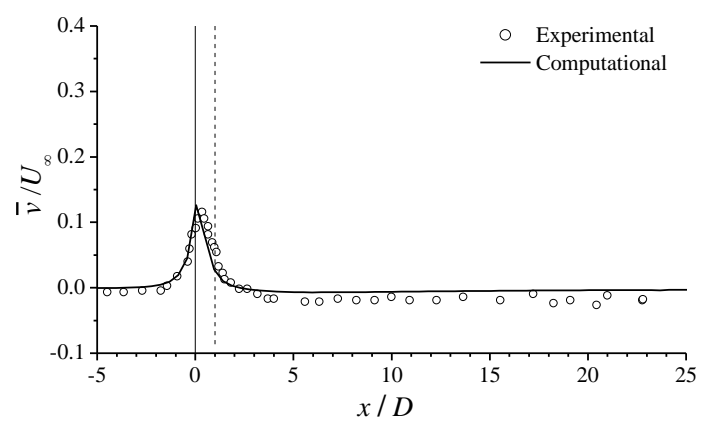

(a) $\varphi=0.03, H=0.133 \mathrm{~m}$

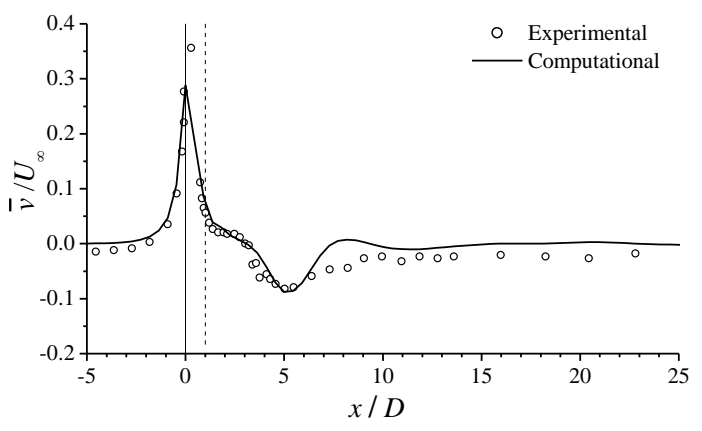

(b) $\varphi=0.10, H=0.133 \mathrm{~m}$

Figure 2 Mean velocity profiles: along $\mathrm{Y}=\mathrm{D} / 2$

\subsection{Turbulent velocity profiles}

As the cylinder Reynolds number, $R e_{d}=588$, is greater than the typical transition value of 190 [4], the characteristics of flow around single cylinders would become three-dimensional. Hence, the turbulent velocity profiles are required to assess the performance of the current model. The intensity of turbulent fluctuations is estimated as the root-mean-square of the fluctuating velocity, $u_{r m s}=\sqrt{\left(u^{\prime}\right)}$ and

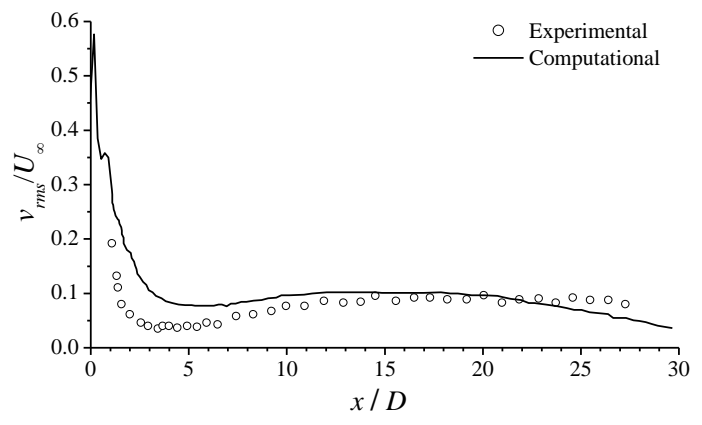

(a) $\varphi=0.03, H=0.133 \mathrm{~m}$

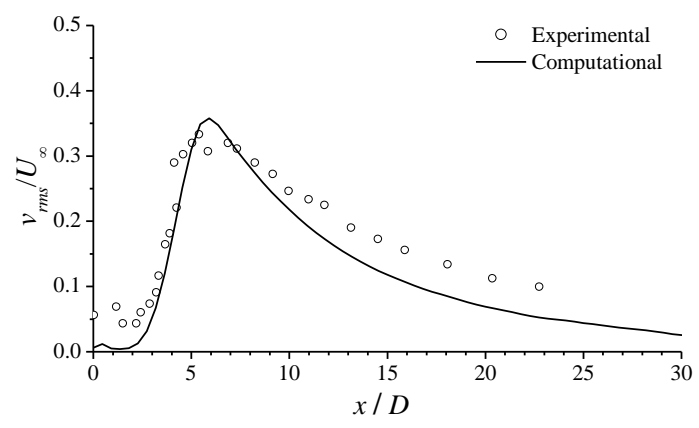

(b) $\varphi=0.10, H=0.133 \mathrm{~m}$

Figure 3 Turbulent velocity profiles: $v_{r m s}$ along centreline $\mathrm{Y}=0$

$v_{r m s}=\sqrt{\overline{\left(v^{\prime}\right)}}$. Estimated $v_{r m s}$ along centreline $Y=0$ from both experimental and computational results are shown in Figure 3. The computed results are consistent with the experimental data in both cases. While the peak in Figure 3(a) indicates that the small-scale flow generated by individual cylinders can be reproduced, the peak in Figure 3(b) occurs at some distance behind the patch, corresponding to the patch-scale vortices of the Karman vortex street.

\section{Results}

\subsection{Velocity field}

Figure 4 shows the contour map of dimensionless mean velocity $\left(\bar{u} / U_{\infty}\right)$ under different densities and water depths. Because of the porous vegetation zone, the flow can pass through it and alter the wake structure. As shown in Figure 1 and Figure 4, the diversion flow begins at a distance of about $D$ 
upstream the patch, and the longitudinal velocity $\bar{u}$ decreases from this region. However, the transverse velocity $\bar{v}$ along $Y=D / 2$ increases and reaches its peak at $X=0$ in Figure 2, meaning that the diversion is the strongest at the leading edge of the patch. A wider region of diversion can be observed in Figure 4(d-f) than that in Figure 4(a-c), and the peak value of $\bar{v}$ in Figure 2(b) is also larger than that in Figure 2(a), demonstrating that a dense patch can enhance the diversion flow. Inside the patch, the diversion flow becomes weaker and the velocity drops rapidly due to drag. Behind the patch, the reduced velocity remains nearly constant over a certain distance. Within this region, both the velocity and turbulence are reduced, relative to the adjacent bare bed.[9] Therefore, this region is called the steady wake region [10]. In general, both the distance of the steady wake region and the reduced velocity in this region decrease as $\varphi$ increases. Behind the steady wake, the velocity $\bar{u}$ begins to increase, recovering toward the free-stream velocity, $U_{\infty}$. The von Karman vortex street can also be found in this region when $0.05<\varphi \leq 0.15$ [11]. For the sparse patch $(\varphi=$ 0.03 ), the distance of steady wake and recovery region doesn't change so much with water depth $H$. But when $\varphi=0.1$, both regions get shorter as $H$ increases.

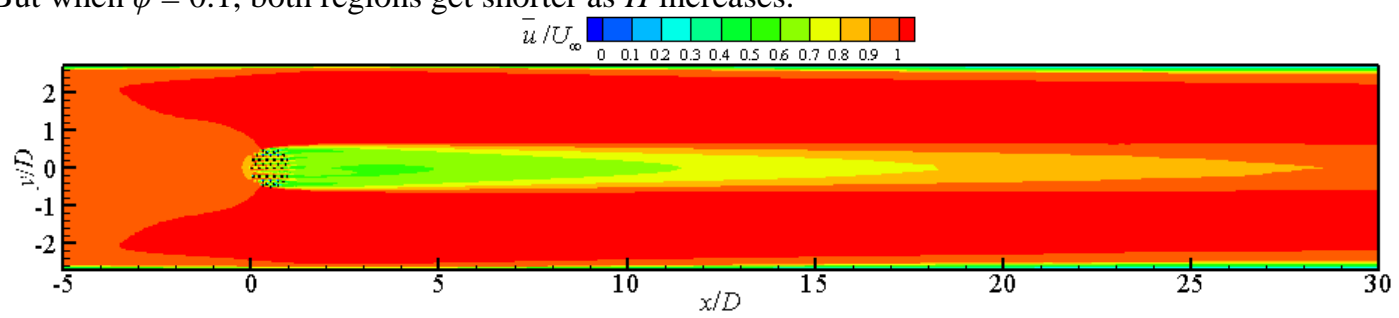

(a) $\varphi=0.03, H=0.0665 \mathrm{~m}$

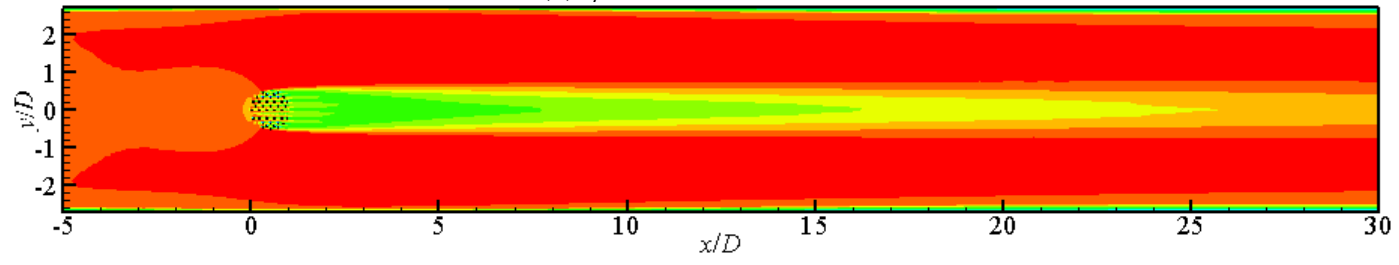

(b) $\varphi=0.03, H=0.133 \mathrm{~m}$

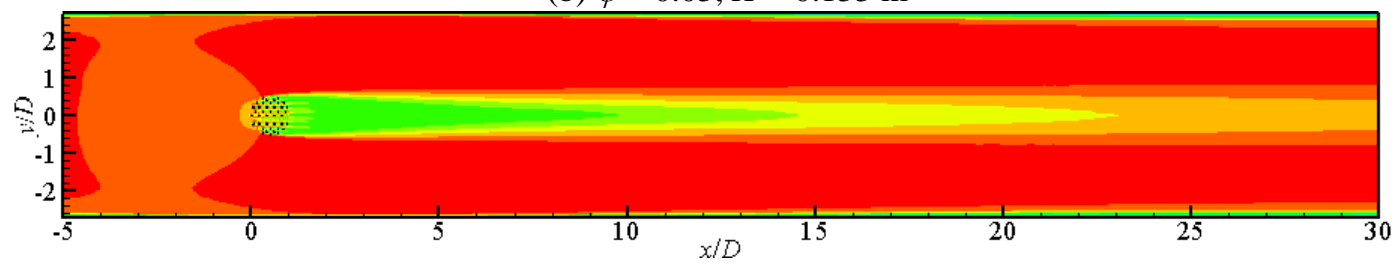

(c) $\varphi=0.03, H=0.266 \mathrm{~m}$

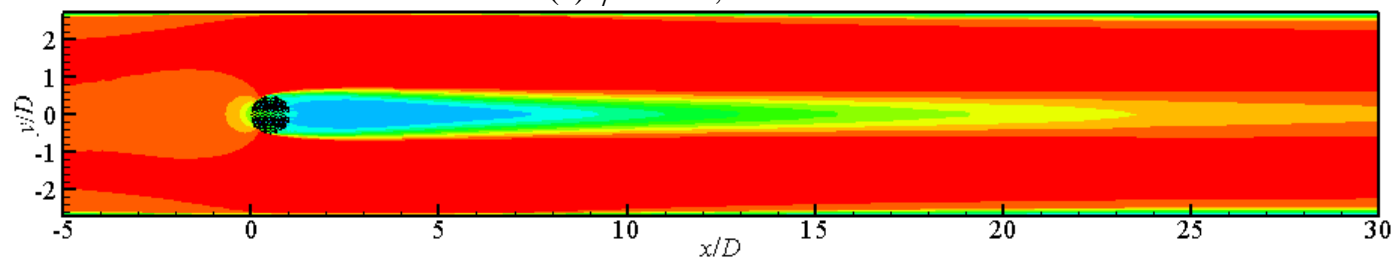

(d) $\varphi=0.10, H=0.0665 \mathrm{~m}$ 


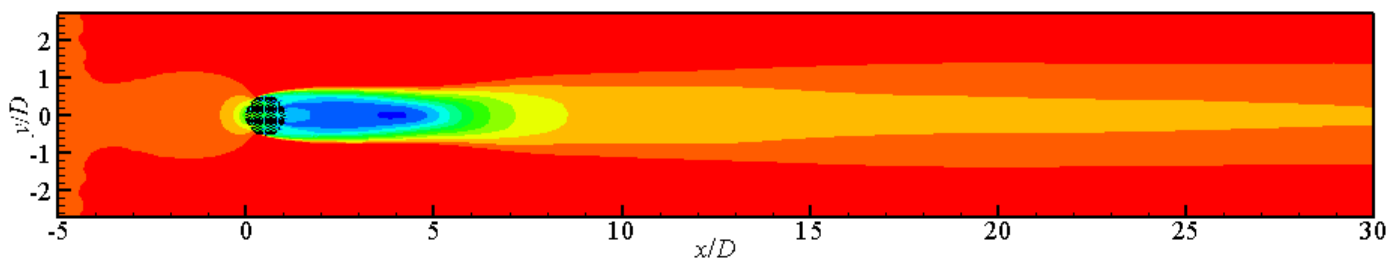

(e) $\varphi=0.03, H=0.133 \mathrm{~m}$

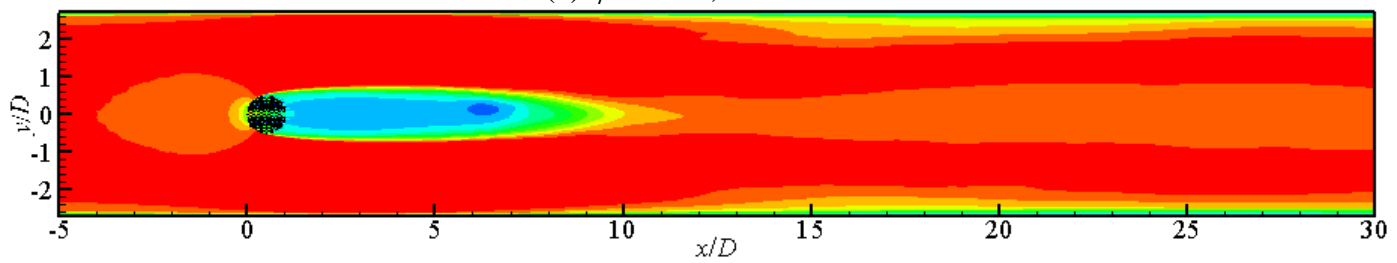

(f) $\varphi=0.10, H=0.266 \mathrm{~m}$

Figure 4 Contour map of dimensionless mean velocity

\subsection{Bed shear stress}

Vegetation can create regions of sediment retention by reducing the flow velocity and shear stress. Although it is not yet clear that whether sediment transport within vegetation is only related to mean bed stress, it is reasonable to expect that bed stress plays a contributing rule[9]. Therefore, it is meaningful and of practical use to analyze the distribution of bed shear stress. In this study, the bed stress, $\tau_{b c}$ is calculated by:

$$
\tau_{b c}=\rho u_{*}^{2}
$$

where $\rho$ is the density of water, $u *$ is the friction velocity, and

$$
u_{*}=k u_{z} / \ln \left(z / z_{0}\right)
$$

$k$ is the von Karman parameter, $u_{\mathrm{z}}$ is the flow velocity at surface $z$ above the bed, $z_{0}$ is the roughness height used in simulations. The dimensionless bed stress, $\tau_{b c} / \tau_{b}$ is used in Figure 5 for comparisons, and $\tau_{b}$ is the bed stress of bare bed without the patch. As shown in Figure 5, the whole channel region can be divided into three different zones: the strengthened zone between the shoulder of the patch and the side wall, the restricted zone in the steady wake and the remaining unaffected zone. For the case $\varphi=0.03$, both the area of the strengthened zone and the value of $\tau_{b c} / \tau_{b}$ in this zone are small. In the restricted zone, the bed stress is strongly restricted as a result of the steady wake, and the length of this zone in $x$-direction depends on the distance of steady wake and recovery region. As $\varphi$ increases to 0.1 , both the strengthened zone and the restricted zone enlarge, the value of bed stress increases in the strengthened zone and decreases in the restricted zone. There is a small zone near the leading edge of the patch, where the bed stress is also weakened because of the diversion flow. Additionally, both the range of the strengthened zone and the magnitude of bed stress in strengthened zone increases with $H$. Therefore, the deposition in the wake behind the patch can be enhanced by increasing $\varphi$.

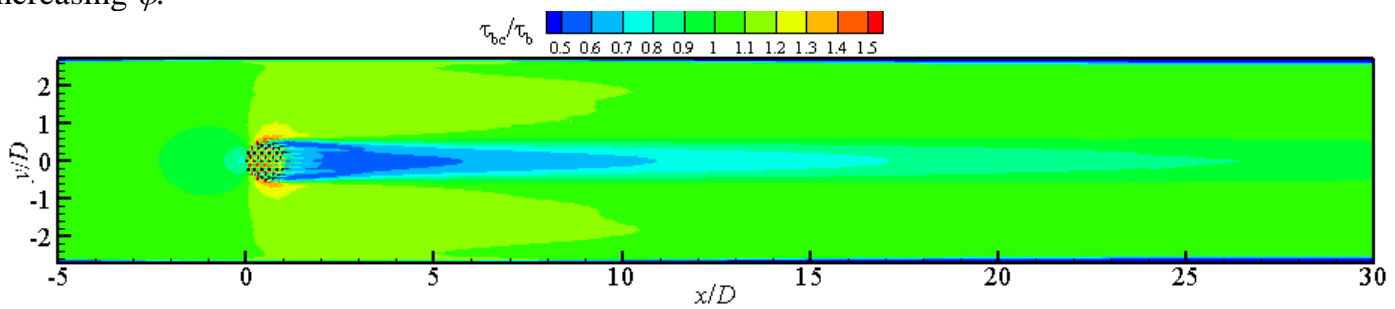

(a) $\varphi=0.03, H=0.0665 \mathrm{~m}$ 


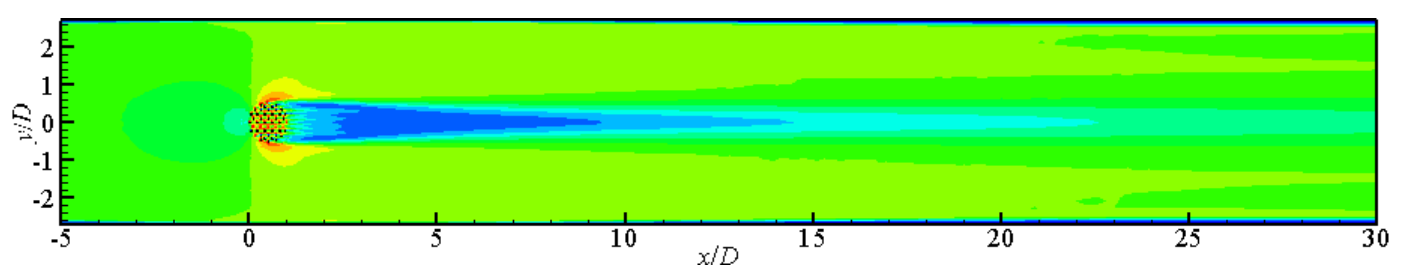

(b) $\varphi=0.03, H=0.133 \mathrm{~m}$

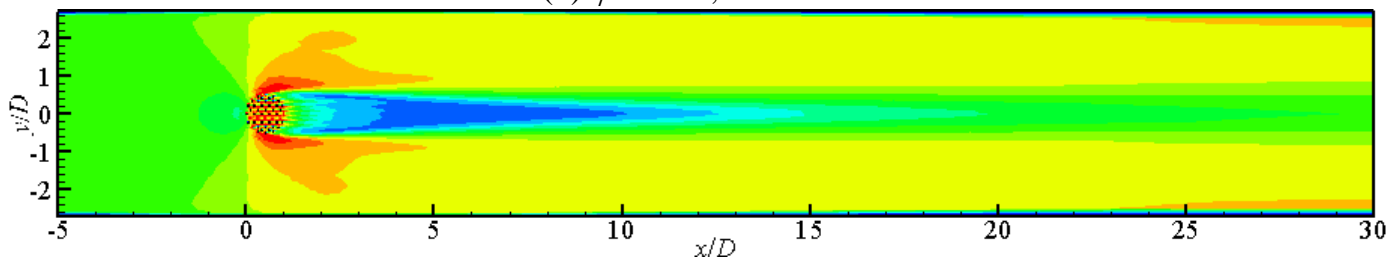

(c) $\varphi=0.03, H=0.266 \mathrm{~m}$

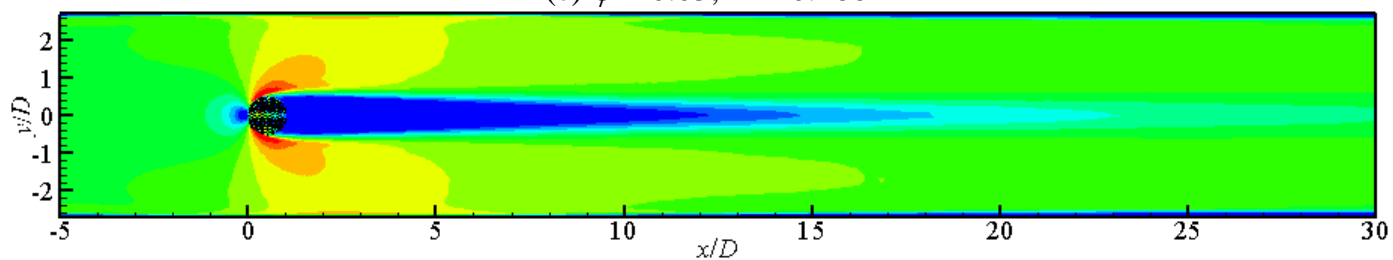

(d) $\varphi=0.10, H=0.0665 \mathrm{~m}$

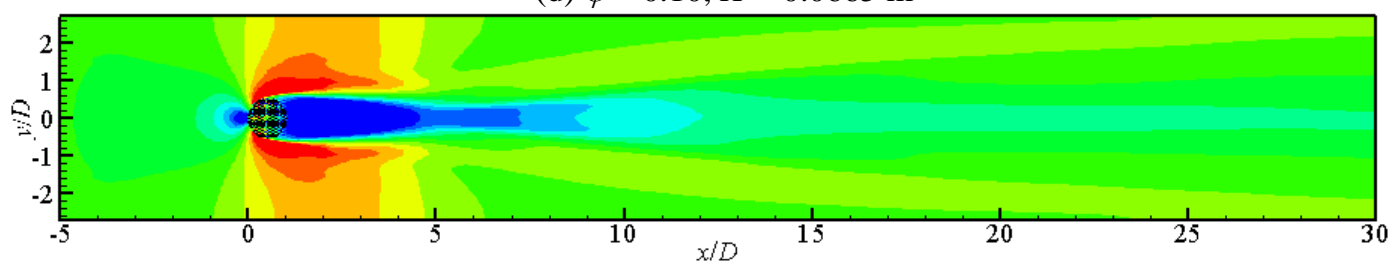

(e) $\varphi=0.10, H=0.133 \mathrm{~m}$

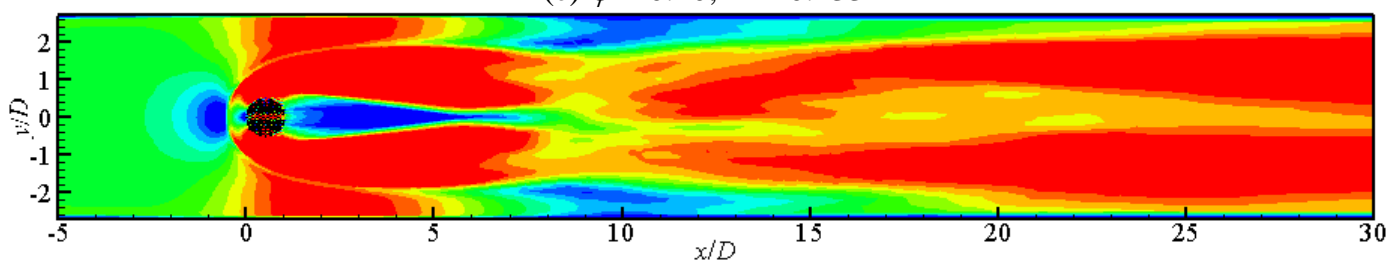

(f) $\varphi=0.10, H=0.266 \mathrm{~m}$

Figure 5 Contour map of dimensionless mean bed shear stress

\section{Conclusions}

In this paper, we present a 3D hydrodynamic model for the shallow water flow through a circular patch of non-submerged cylinders. This model adopts the SST k $-\omega$ turbulence model and considers the moving free surface. Six study cases with different solid volume fractions of the patch and water depths are simulated. The computed results are validated by the experimental data, and good agreements are shown in both the mean and turbulent velocity profiles. The model not only accurately predicts the patch-scale flow patterns, but also reproduces the small-scale flow generated by individual cylinders. The velocity and bed stress field of results are also analyzed and discussed. As a 
conclusion, the density of vegetation is an important factor that alters the flow structure. The influence of water depth on steady wake is small at low density. What's more, the bed stress in steady wake can be reduced greatly with dense patch, which is beneficial for deposition. The bed stress in strengthened zone increases when water depth increases, which may cause more erosion.

\section{Acknowledgements}

The research is supported by China Scholarship Council.

\section{References}

[1] I Nezu, K Onitsuka, Turbulent structures in partly vegetated open-channel flows with LDA and PI V measurements, Journal of hydraulic research, 2001, 39(6): 629-42.

[2] M Ghisalberti, HM Nepf, Mixing layers and coherent structures in vegetated aquatic flows, Journal of Geophysical Research: Oceans, 2002, 107(C2):

[3] J-m Zhan, W-q Hu, W-h Cai, et al., Numerical simulation of flow through circular array of cylinders using porous media approach with non-constant local inertial resistance coefficient, Journal of Hydrodynamics, Ser B, 2017, 29(1): 168-71.

[4] LH Yu, JM Zhan, YS Li, Numerical Simulation of Flow through Circular Array of Cylinders Using Multi-Body and Porous Models, Coast Eng J, 2014, 56(3):

[5] LJ Zong, H Nepf, Vortex development behind a finite porous obstruction in a channel, Journal of Fluid Mechanics, 2012, 691(368-91.

[6] V Casulli, GS Stelling, Numerical simulation of 3D quasi-hydrostatic, free-surface flows, J Hydraul Eng-Asce, 1998, 124(7): 678-86.

[7] NA Phillips, A coordinate system having some special advantages for numerical forecasting, Journal of Meteorology, 1957, 14(2): 184-5.

[8] RM FLORIAN, Zonal two equation turbulence models for aerodynamic flows, AIAA Paper 2906, 1993, 93.

[9] HM Nepf, Hydrodynamics of vegetated channels, Journal of Hydraulic Research, 2012, 50(3): 262-79.

[10] DJ Ball, N Allison, PK Stansby, Modeling shallow water flow around pile groups, Ice Proceedings Water Maritime \& Energy, 1996, 118(4): 226-36.

[11] A Nicolle, I Eames, Numerical study of flow through and around a circular array of cylinders, Journal of Fluid Mechanics, 2011, 679(7): 1-31. 